Poincare Journal of Analysis E Applications

Vol. 2017 (1), 35-42

(C) Poincare Publishers

\title{
Markov moment problems in concrete spaces
}

\author{
OCTAV OLTEANU
}

\author{
Date of Receiving : $\quad$ : 16.03 .2017 \\ Date of Revision : : $\quad 01.06 .2017$ \\ Date of Acceptance : $\quad 23.06 .2017$
}

\begin{abstract}
In the first part of this work a necessary and sufficient condition and respectively a sufficient condition for the existence of a solution of a concrete Markov moment problem are given. The latter result also yields the uniqueness of the solution. Both these two results are based on previous theorems on the abstract moment problem, applied to concrete spaces. Namely, the space which the solution is defined on is a space of analytic functions of several variables, while the target space is an order complete Banach lattice of selfadjoint operators (the bicommutant). The purpose of the second part is to continue the previous study of the author on inverse problems related to the multidimensional Markov moment problem.
\end{abstract}

\section{Introduction}

The classical moment problem is an interpolation problem with a constraint on the positivity of the (linear) solution. Usually, the representation of the solution by means of a positive scalar or vector measure is pointed out. The Markov moment problem involves an additional upper constraint. This constraint controls the norm of the solution. In case of representation of the solution as an element $h$ of an $L^{\infty}$ - space, the two constraints mentioned above lead to the conclusion $0 \leq h \leq b$ a.e., where $\mathrm{b}$ is a positive real constant which appears naturally from a computation. All the aspects on the classical moment problem are strongly related to the form of positive polynomials on the set under discussion [1], [2], [5], [23], [24], [29], [31], [33]. For the classical moment problem (respectively Markov moment problem) see [1] (respectively [11]). Meanwhile, connections of the moment problem with some other fields have been done [4]. In the latter work, elements of fixed point theory related to the moment problem are applied. For operator valued moment problems see [16] - [19], [33]. Some other connections to operator theory are revealed in [29], [33]. The latter work contains also solutions of moment problems on unbounded subsets. Three main aspects are studied in the moment problem: existence, uniqueness and construction of the solution. The first part of the present work focuses on the existence problem. One gives necessary

2010 Mathematics Subject Classification. 47A57, 46E10, 45Q05.

Key words and phrases. Markov moment problem, concrete spaces, inverse problems.

Communicated by: Henri Bonnel 